\title{
Reprocessing of Soft X-ray Emission Lines in Black Hole Accretion Disks
}

Christopher W. Mauche, Duane A. Liedahl, Benjamin F. Mathiesen, Mario A. Jimenez-Garate, and John C. Raymond

This article to appear in The Astrophysical Journal

\section{January 5, 2004}

U.S. Department of Energy

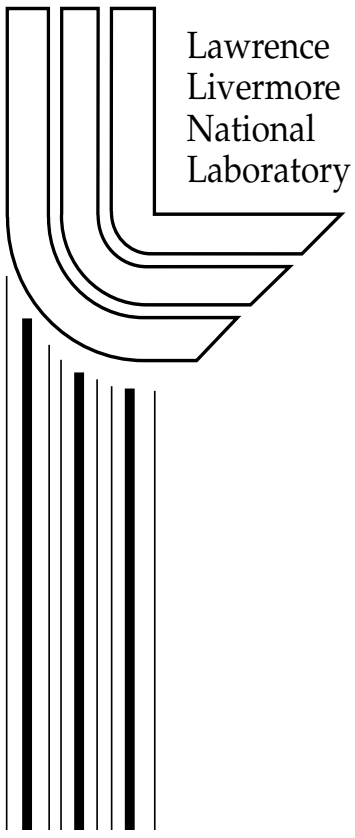




\section{DISCLAIMER}

This document was prepared as an account of work sponsored by an agency of the United States Government. Neither the United States Government nor the University of California nor any of their employees, makes any warranty, express or implied, or assumes any legal liability or responsibility for the accuracy, completeness, or usefulness of any information, apparatus, product, or process disclosed, or represents that its use would not infringe privately owned rights. Reference herein to any specific commercial product, process, or service by trade name, trademark, manufacturer, or otherwise, does not necessarily constitute or imply its endorsement, recommendation, or favoring by the United States Government or the University of California. The views and opinions of authors expressed herein do not necessarily state or reflect those of the United States Government or the University of California, and shall not be used for advertising or product endorsement purposes.

This is a preprint of a paper intended for publication in a journal or proceedings. Since changes may be made before publication, this preprint is made available with the understanding that it will not be cited or reproduced without the permission of the author.

\section{AUSPICES STATEMENT}

This work was performed under the auspices of the U.S. Department of Energy by University of California, Lawrence Livermore National Laboratory under Contract W-7405-Eng-48. 


\title{
REPROCESSING OF SOFT X-RAY EMISSION LINES IN BLACK HOLE ACCRETION DISKS
}

\author{
Christopher W. Mauche, Duane A. Liedahl, Benjamin F. Mathiesen \\ Lawrence Livermore National Laboratory, 7000 East Avenue, Livermore, CA 94550; \\ mauche@cygnus.llnl.gov, liedahl1@1ln1.gov, mathiesen2@1lnl.gov \\ Mario A. Jimenez-GARATE \\ Massachusetts Institute of Technology, Center for Space Research, 70 Vassar Street, Cambridge, MA 02139; \\ mario@space.mit.edu \\ AND \\ JOHN C. RAYMOND \\ Harvard-Smithsonian Center for Astrophysics, 60 Garden Street, Cambridge, MA 02138; jraymond@cfa.harvard.edu \\ Re-submitted to ApJ Letters 2004 January 5
}

\begin{abstract}
By means of a Monte Carlo code that accounts for Compton scattering and photoabsorption followed by recombination, we have investigated the radiation transfer of $\operatorname{Ly} \alpha, \operatorname{He} \alpha$, and recombination continua photons of $\mathrm{H}$ - and He-like $\mathrm{C}, \mathrm{N}, \mathrm{O}$, and Ne produced in the photoionized atmosphere of a relativistic black hole accretion disk. We find that photoelectric opacity causes significant attenuation of photons with energies above the O VIII K-edge; that the conversion efficiencies of these photons into lower-energy lines and recombination continua are high; and that accounting for this reprocessing significantly (by factors of $21 \%$ to $105 \%$ ) increases the flux of the $\operatorname{Ly} \alpha$ and $\mathrm{He} \alpha$ emission lines of $\mathrm{H}$ - and $\mathrm{He}$-like $\mathrm{C}$ and $\mathrm{O}$ escaping the disk atmosphere.
\end{abstract}

Subject headings: accretion, accretion disks — black hole physics — galaxies: individual (MCG -6-30-15) — galaxies: Seyfert — radiative transfer $-\mathrm{X}$-rays: galaxies

\section{INTRODUCTION}

A controversy currently exists concerning the interpretation of the broad spectral features observed in the soft X-ray spectra of type 1 Seyfert galaxies. Lee et al. (2001) and Turner et al. (2003) interprete the features seen in the Chandra High Energy Transmission Grating (HETG) and XMM-Newton Reflection Grating Spectrometer (RGS) spectra of MCG -6-30-15 as absorption edges produced by a dusty, partially ionized ("warm") absorber, while Branduardi-Raymont et al. (2001), Mason et al. (2003), and Sako et al. (2003) interpret the features seen in the XMM-Newton RGS spectra of MCG -6-30-15 and Mrk 766 as relativistically broadened and gravitationally redshifted $\mathrm{Ly} \alpha$ emission lines of $\mathrm{H}$-like $\mathrm{C}, \mathrm{N}$, and $\mathrm{O}$ arising in the accretion disk surrounding a maximally spinning Kerr black hole. While warm absorbers are a common feature of Seyfert 1 galaxies, the relativistic line interpretation is appealing because it produces model parameters (disk inclination, emissivity index, and inner radius) that are not inconsistent with those derived for the $\mathrm{Fe}$ $\mathrm{K} \alpha$ line.

To be consistent with observations, proponents of the relativistic line interpretation must explain the apparent absence of the $\operatorname{Ly} \beta$ lines and radiative recombination continua (RRCs) of $\mathrm{C}, \mathrm{N}$, and $\mathrm{O}$, as well as emission by higher- $Z$ ions. BranduardiRaymont et al. (2001) and Sako et al. (2003) propose that this is accomplished by radiation transfer effects, specifically that the higher-order Lyman lines are suppressed by line opacity, while the $\mathrm{C}, \mathrm{N}$, and $\mathrm{O}$ RRCs and the higher- $Z$ emission features are suppressed by photoelectric opacity. The production of $\mathrm{C}, \mathrm{N}$, and $\mathrm{O}$ emission lines in photoionized constant-density and hydrostatic disk models has been studied quantitatively by Ballantyne, Ross, \& Fabian (2002, hereafter BRF). These calculations do not, however, account for the reprocessing of soft $\mathrm{X}$-ray photons into lower-energy lines and RRCs. In a moderately ionized solar abundance plasma, the continuum opacity is dominated by $\mathrm{C}$ from 0.39 to $0.74 \mathrm{keV}$ and $\mathrm{O}$ from 0.74 to
$2.4 \mathrm{keV}$ (Fig. 1). If, for example, the medium is optically thick to photoelectric absorption by $\mathrm{O}^{7+}$, a fraction of the photons with energies greater than that of the $\mathrm{O}$ VIII K-edge will be absorbed, and a fraction of those will be reradiated as O VIII Ly $\alpha$ photons, thereby increasing the equivalent width of the line. To explore such processes quantitatively, we have combined a disk atmosphere code with a Monte Carlo radiation transfer code to calculate the emergent spectrum of the irradiated atmosphere of a relativistic black hole accretion disk. In this first communication, we concentrate on the physics of the radiation transfer of $\mathrm{Ly} \alpha, \mathrm{He} \alpha$, and recombination continua photons of $\mathrm{H}-$ and $\mathrm{He}-$ like $\mathrm{C}, \mathrm{N}, \mathrm{O}$, and $\mathrm{Ne}$, leaving to a future work the calculation of equivalent widths and a detailed comparison with data.

\section{DISK ATMOSPHERE AND RADIATION TRANSFER CODES}

The disk atmosphere code calculates the structure of the atmosphere of a black hole accretion disk photoionized by an external source of nonthermal X-rays. The code is adapted from that used by Jimenez-Garate, Raymond, \& Liedahl (2002) to model the accretion disk atmospheres of X-ray binaries. It assumes axial symmetry and solves the hydrostatic balance, photoionization equilibrium, and radiation transfer equations on an adaptive mesh in the slab approximation in a succession of decoupled disk annuli using the Raymond (1993) photoionization plasma code, which includes the X-ray emissivities and opacities of all ions of the 12 most abundant elements. The disk is assumed to have solar abundances, to be gas-pressure dominated, and to have a structure given by Shakura \& Sunyaev (1973) with the scale radius $r_{*}=r_{g}=G M / c^{2}$, modified by the Page \& Thorne (1974) and Riffert \& Herold (1995) general relativistic corrections to the vertical structure. We assume that the incident angle of the nonthermal radiation $i=55^{\circ}$, to simulate isotropic illumination, and that the ratio of the incident nonthermal flux to the disk thermal flux $f=\int F_{X} d \nu / \int F_{\text {disk }} d \nu=1$. The lower boundary conditions of the calculation are the disk 
temperature $\left[T_{\text {disk }}(r) \propto r^{-3 / 4}\right]$ and pressure, while the upper boundary condition is the Compton temperature, which is reduced to $T_{C} \sim 10^{7} \mathrm{~K}$ by the thermal disk emission that irradiates the atmosphere from below.

As with LMXB disks, the structure of the atmosphere of an AGN disk is sensitive to the assumptions about the behavior of photoionized plasma on the unstable branches of the thermal equilibrium S-shaped curve (Krolik, McKee, \& Tarter 1981). To maximize the production of soft X-ray lines from H-like ions, we assume thermal equilibrium solutions on the upper branch of the S-shaped curve. Furthermore, we ignore the effects of the radiation pressure in the hydrostatic balance equation, since it reduces the thickness of the atmosphere and the production of soft X-ray lines, and since in a real disk dynamical and magnetic pressures may counteract such a reduction (Miller \& Stone 2000). We assume that the nonthermal continuum has a power-law shape with a photon index $\Gamma=2.1$ with a high-energy exponential cutoff $E_{\text {high }}=150 \mathrm{keV}$ and a low-energy exponential cutoff $E_{\text {low }}=k T_{\text {disk }}$; the black hole has mass $M=10^{7} \mathrm{M}_{\odot}$ and spin $a=0.998$, so the radius of the innermost stable circular orbit $r_{\mathrm{ISCO}}=1.23 r_{g}$ (Thorne 1974); the accretion rate $\dot{M}=10^{24} \mathrm{~g} \mathrm{~s}^{-1}=0.2 \dot{M}_{\mathrm{Edd}}$; and the viscosity parameter $\alpha=0.01$. The model has $499 \hat{z} \times 35 \hat{r}=$ $17.5 \times 10^{3}$ cells, with the annular radii $r=1600 k^{-2} r_{g}$, with $k=2,3,4, \ldots, 36$. For additional details about the disk atmosphere model, see Jimenez-Garate et al. (2003).

While the radiation transfer equation used in the disk atmosphere code is sufficiently accurate to calculate the disk atmosphere structure, it cannot be used to calculate its spectrum. To solve this problem, we are developing a Monte Carlo code that accounts for radiation transfer in the atmosphere of a relativistic black hole accretion disk. On a microphysical scale, we assume that photons are subject to Compton and photoelectric opacity. For a photon of energy $E$ scattering off an electron drawn from a population with an isotropic Maxwellian velocity distribution with temperature $k T$, the Compton cross section

$$
\sigma_{C}=\sigma_{T}\left[1-2\left(\frac{E}{m_{e} c^{2}}\right)+\frac{26}{5}\left(\frac{E}{m_{e} c^{2}}\right)^{2}\left(1+\frac{k T}{m_{e} c^{2}}\right)\right],
$$

where $\sigma_{T}$ is the Thomson cross section, which is valid for $E \ll$ $m_{e} c^{2}$ and $k T \ll m_{e} c^{2}$ (Rybicki \& Lightman 1979). For a photon with energy $E$ and direction vector $\hat{p}$ scattering off an electron with velocity $\beta c, \gamma=\left(1-\beta^{2}\right)^{-1 / 2}$, and direction vector $\hat{e}$, the scattered photon energy $E^{\prime}=\Pi^{\prime}(0) / c$ and direction vector $\hat{p}^{\prime}=\Pi^{\prime}(1: 3) / \Pi^{\prime}(0)$. In these expressions, the scattered photon 4-momentum $\Pi^{\prime}=R_{e}^{-1} \Lambda^{-1} R_{\gamma}^{-1} S(\Delta \theta) R_{\gamma} \Lambda R_{e} \Pi$, where $R_{e}$ and $R_{\gamma}$ are the electron and photon rotation matrices, respectively; $\Lambda$ is the Lorentz transformation matrix; and $S(\Delta \theta)=$ $Q R(\Delta \theta)$ is the scattering matrix. In this expression, $Q=$ $1 /\left[1+\gamma E(1-\beta \hat{e} \cdot \hat{p})(1-\cos \Delta \theta) / m_{e} c^{2}\right]$ and the scattering angle $\Delta \theta$ is given by the Klein-Nishina formula for the differential Compton scattering cross section. The photoelectric opacity is calculated at each energy for 446 subshells of 140 ions of the 12 most abundant elements of a solar abundance plasma using the partial photoionization cross sections of Verner \& Yakovlev (1995). Following photoabsorption by K-shell ions, we generate RRC and recombination line cascades in a probabilistic manner using the recombination cascade calculations described by Sako et al. (1999). The shapes of the RRCs are determined by the functional form of the photoionization cross sections and the local electron temperature. Recombination emission from L-shell ions is ignored, since it is suppressed by resonant Auger destruction (Ross, Fabian, \& Brandt 1996).
On a macrophysical scale, we assume that the velocity of the gas is restricted to the azimuthal direction and has a value $v_{\phi}=v_{K} /\left[1+\left(v_{K} / c\right)^{3}\right]$, where $v_{K}=\sqrt{G M / r}$ is the Keplerian value, as appropriate for a maximally spinning Kerr black hole (Bardeen, Press, \& Teukolsky 1972); that within each cell the gas moves with a uniform velocity; and that photons travel in straight lines. By imposing a limit on the velocity difference from one cell to the next, we determine the radial and azimuthal dimensions of the cells used in the Monte Carlo calculation. Specifically, we require that $\Delta v<2 \times 10^{8} \mathrm{~cm} \mathrm{~s}^{-1}$ [set by $\Delta v=\Delta \lambda c / \lambda$ with $\lambda=1.78 \AA$ (Fe XXVI Ly $\alpha$ ) and $\Delta \lambda=0.012 \AA$ (the Chandra High Energy Grating FWHM)]. Extending the grid from $r=1.23 r_{g}$ to $600 r_{g}$, the model has $499 \hat{z} \times 108 \hat{r} \times 52-1005 \hat{\phi}=21.5 \times 10^{6}$ cells.

Photons are propagated in this grid until they are destroyed or escape. From the "event list" of escaping photons, we can calculate the emergent spectrum in the fluid frame, in the local inertial frame (accounting for Lorentz boosts), and in the inertial frame at infinity (accounting for gravitational redshifts). However, because we do not yet follow photons along geodesics, we are not yet able to directly calculate the observed spectrum at infinity at a given disk inclination. To solve this problem, we extracted from the Laor (1991) model in XSPEC the line profiles $L_{k}(\nu)$ and relative weights $w_{k}$ for a $\delta$-function spectrum for each disk annulus for an inclination angle $i=35^{\circ}$, as typical of Seyfert 1 galaxies. The observed spectrum at infinity at this inclination in the Kerr metric is then $S^{\prime}\left(\nu_{i}\right)=$ $\sum_{k} w_{k} \sum_{j} S_{k}\left(\nu_{j}\right) L_{k}\left(\nu_{i}, \nu_{j}\right)$, where $S_{k}(\nu)$ are the spectra in the fluid frame for each disk annulus.

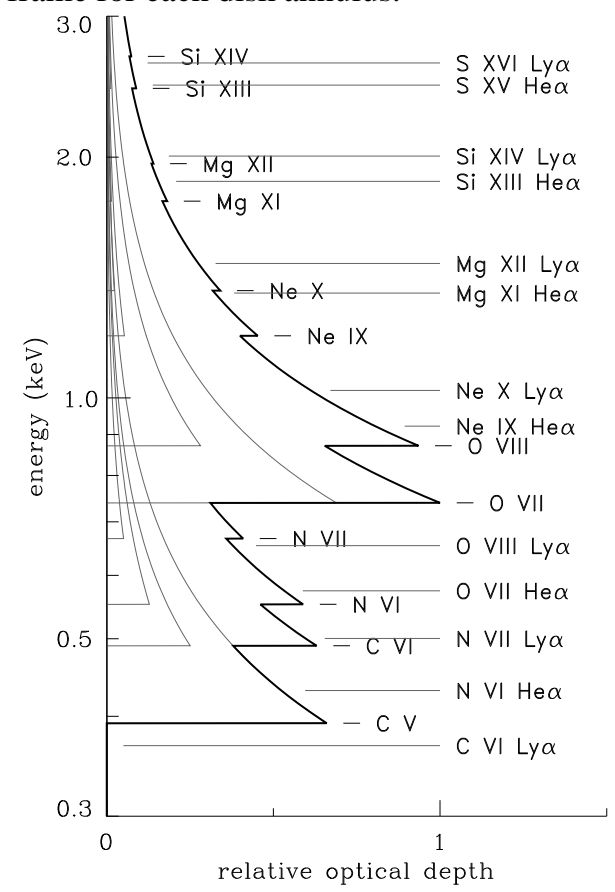

FIG. 1.- Energy dependence of the optical depth to photoelectric absorption of a solar abundance plasma with unit ionization fractions of $\mathrm{H}$ - and He-like $\mathrm{C}$, $\mathrm{N}, \mathrm{O}, \mathrm{Ne}, \mathrm{Mg}$, and Si. Energies of the $\mathrm{Ly} \alpha(\mathrm{He} \alpha)$ lines of $\mathrm{H}$-like (He-like) C, $\mathrm{N}, \mathrm{O}, \mathrm{Ne}, \mathrm{Mg}, \mathrm{Si}$, and $\mathrm{S}$ are indicated. Note that $\mathrm{C}(\mathrm{O})$ dominates the opacity from 0.39 to 0.74 (0.74 to 2.4$) \mathrm{keV}$.

\section{MONTE CARLO SPECTRA}

The Monte Carlo code can be configured to run in a number of ways, but for the present investigation we used it to study the radiation transfer of recombination photons produced in the 
disk atmosphere $\left(\tau_{T} \leq 10\right)$. For each transition, the emissivities $j_{Z, i}=n_{e} n_{p} A_{Z} f_{Z, i+1} \alpha T^{-\beta}$ photons $\mathrm{cm}^{-3} \mathrm{~s}^{-1}$, where $n_{e}$ is the electron density, $n_{p}$ is the proton density, $A_{Z}$ is the abundance of element $Z$ relative to $\mathrm{H}, f_{Z, i+1}$ is the ionization fraction of the $i+1$ charge state of element $Z$, and $\alpha T^{-\beta}$, is the temperature-dependent "per-ion" recombination rate coefficient based on power-law fits to the recombination rates over the temperature range of interest. Because the recombination luminosity of our disk atmosphere model is dominated by low- $Z$ ions, we performed Monte Carlo calculations for the $n=2 \rightarrow 1$ (Ly $\alpha$ doublets and $\operatorname{He} \alpha$ triplets) and RRC transitions of $\mathrm{H}$ - and He-like $\mathrm{C}, \mathrm{N}, \mathrm{O}$, and $\mathrm{Ne}$. In addition, we performed calculations for the $\mathrm{Ly} \alpha$ doublets of $\mathrm{H}$-like $\mathrm{Mg}, \mathrm{Si}$, $\mathrm{S}, \mathrm{Ar}, \mathrm{Ca}$, and $\mathrm{Fe}$ and for the brightest line of the brightest $\mathrm{Fe}$ L-shell ion (Fe XVII $\lambda 17.05 \AA$ ), but found for the adopted disk model that they have only a small effect on the net spectrum.
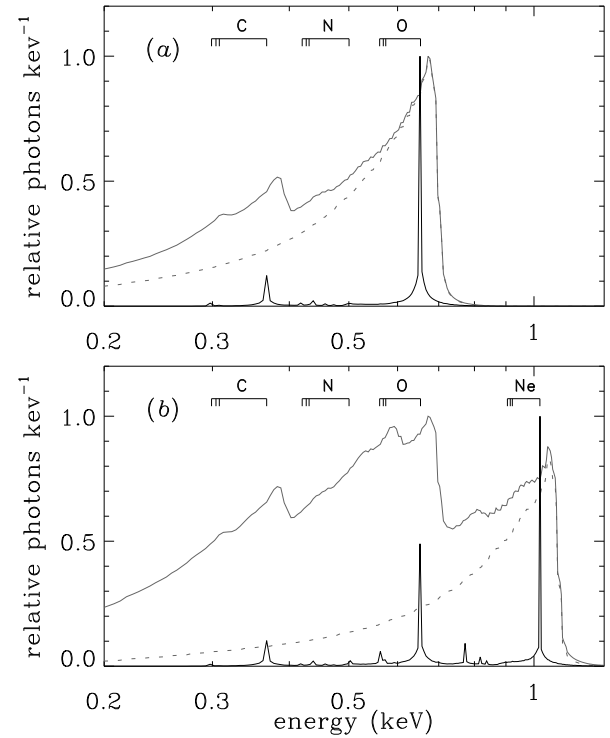

FIG. 2.- Emergent spectra resulting from the production in the disk of Ly $\alpha$ photons of $\mathrm{H}$-like $\mathrm{O}(a)$ and $\mathrm{Ne}(b)$. Black curves are the spectra in the fluid frame, solid gray curves are the spectra at infinity at $i=35^{\circ}$, and the dotted gray curves are the spectra ignoring recombination following photoionization. Energies of various $\operatorname{Ly} \alpha$ and $\operatorname{He} \alpha$ lines are indicated.

Figure 2 shows the emergent spectra resulting from the production in the disk of Ly $\alpha$ photons of H-like $\mathrm{O}$ and $\mathrm{Ne}$. In the upper panel, $80 \%$ of the escaping photons are in the O VIII Ly $\alpha$ line and $9 \%$ are in the C VI Ly $\alpha$ line. In the lower panel, $42 \%$ of the escaping photons are in the $\mathrm{Ne} X \mathrm{Ly} \alpha$ line, 26\% are in the $\mathrm{O}$ VIII Ly $\alpha$ line, and $5 \%$ are in the $\mathrm{C}$ VI Ly $\alpha$ line. The conversion efficiency of $\mathrm{Ne} \mathrm{X} \operatorname{Ly} \alpha$ into $\mathrm{O}$ VIII Ly $\alpha$ is so high because the former transition lies just above the O VIII K-edge (Fig. 1). In both cases, very little flux escapes in the $\mathrm{N}$ recombination lines because the $\mathrm{C}$ and $\mathrm{O}$ opacities dominate over the $\mathrm{N}$ opacity, and $\mathrm{N}$ recombination lines are subsequently converted into $\mathrm{C}$ recombination lines.

Figure 3 shows the emergent spectra resulting from the production in the disk of RRC photons of $\mathrm{H}$-like $\mathrm{O}$ and $\mathrm{Ne}$. In the upper panel, $39 \%$ of the escaping photons are in the O VIII $\mathrm{RRC}, 30 \%$ are in the $\mathrm{O}$ VIII Ly $\alpha$ line, and $6 \%$ are in the $\mathrm{C} \mathrm{VI}$ Ly $\alpha$ line. In the lower panel, $52 \%$ of the escaping photons are in the $\mathrm{Ne} X \mathrm{RRC}, 4 \%$ are in the $\mathrm{Ne} \mathrm{X} \mathrm{Ly} \alpha$ line, $16 \%$ are in the $\mathrm{O}$ VIII Ly $\alpha$ line, and $3 \%$ are in the $\mathrm{C}$ VI Ly $\alpha$ line. The $\mathrm{O}$ VIII RRC conversion efficiency is so high because the RRC photons are emitted just above the $\mathrm{O}$ VIII K-edge, where the opacity is the highest. The conversion efficiency of Ne X RRC photons into the $\mathrm{Ne} \mathrm{X} \operatorname{Ly} \alpha$ line is so low because the $\mathrm{O}$ opacity dominates over the Ne opacity at the Ne X K-edge (Fig. 1), and because $\mathrm{Ne} X \mathrm{Ly} \alpha$ photons are subsequently converted into the $\mathrm{O}$ VIII Ly $\alpha$ line with high efficiency (Fig. 2b). Once again, the efficiency of conversion into escaping $\mathrm{N}$ recombination lines is very low.
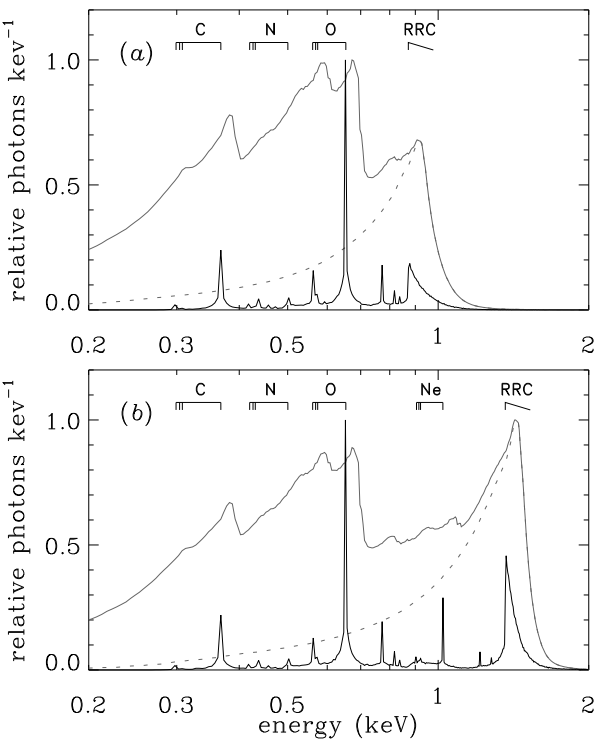

FIG. 3.- Similar to Figure 2, for the RRC photons of H-like $\mathrm{O}(a)$ and $\mathrm{Ne}$ (b). Energies of various $\mathrm{Ly} \alpha$ and $\mathrm{He} \alpha$ lines and the RRCs are indicated.

As these examples demonstrate, recombination following photoionization reprocesses soft X-ray photons into lowerenergy lines and RRCs, and in the process increases the strengths and hence the equivalent widths of the $\mathrm{C}$ and $\mathrm{O}$ emission features. As shown in Table 1, for the adopted disk model, the efficiencies of conversion of the $\operatorname{Ly} \alpha, \operatorname{He} \alpha$, and RRC photons of $\mathrm{H}$ - and He-like $\mathrm{C}, \mathrm{N}, \mathrm{O}$, and $\mathrm{Ne}$ into lower-energy lines and RRCs range from $20 \%$ (for O VIII Ly $\alpha$ ) to $86 \%$ (for Ne IX $\operatorname{He} \alpha)$.

TABLE 1

Conversion Efficiencies of Escaping Photons

\begin{tabular}{lcccc}
\hline \hline Transition & $\mathrm{C}$ & $\mathrm{N}$ & $\mathrm{O}$ & $\mathrm{Ne}$ \\
\hline H-like Ly $\alpha \ldots \ldots$ & $\ldots$ & $36 \%$ & $20 \%$ & $58 \%$ \\
He-like He $\alpha \ldots \ldots$ & $\ldots$ & $30 \%$ & $39 \%$ & $86 \%$ \\
H-like RRC ...... & $41 \%$ & $54 \%$ & $63 \%$ & $72 \%$ \\
He-like RRC .... & $34 \%$ & $33 \%$ & $61 \%$ & $48 \%$ \\
\hline
\end{tabular}

To show the net effect of these processes on the observed spectrum, we show in Figure 4 the full disk atmosphere spectrum accounting for the $n=2 \rightarrow 1$ and RRC transitions of $\mathrm{H}$ - and He-like $\mathrm{C}, \mathrm{N}, \mathrm{O}$, and $\mathrm{Ne}$, with relative normalizations determined by the integrated disk atmosphere emissivities. In this model, the emission spectrum is dominated by $\mathrm{C}, \mathrm{N}$, and $\mathrm{O}$, so the inclusion of $\mathrm{Ne}$ (and higher- $Z$ ions) makes only a minor contribution to the net soft X-ray spectrum. The emergent spectrum is shown two ways in Figure 4. In the upper panel, recombination following photoionization is ignored, while in the lower panel this process is fully accounted for. These spectra differ in two important ways. First, the lower spectrum is brighter than the upper spectrum by $50 \%$ : by accounting for recombination following photoionization, significantly more line flux escapes the disk atmosphere. Specifically, for the adopted disk model, the flux and hence the equivalent widths of the $\mathrm{C} \mathrm{V}$ $\mathrm{He} \alpha$, C VI Ly $\alpha, \mathrm{O}$ VII $\mathrm{He} \alpha$, and O VIII Ly $\alpha$ lines increase 
by $21 \%, 105 \%, 28 \%$, and $44 \%$, respectively. Second, the relative line strengths change: the $\mathrm{C}$ VI $\operatorname{Ly} \alpha$ to $\mathrm{C}$ V He $\alpha$ line ratio increases by $69 \%$, the $\mathrm{O}$ VIII Ly $\alpha$ to $\mathrm{O}$ VII $\mathrm{He} \alpha$ line ratio increases by $12 \%$, and the summed $\mathrm{C} / \mathrm{O}$ line ratio increases by $16 \%$. In both cases, the $\mathrm{N}$ emission features are weak because the $\mathrm{N}$ emissivities are relatively low and little $\mathrm{O}$ and $\mathrm{Ne}$ flux is reprocessed into $\mathrm{N}$ recombination features (note that the emission feature at $0.5 \mathrm{keV}$ in Fig. 4 is a combination of $\mathrm{N}$ VII Ly $\alpha$ and the $\mathrm{C}$ VI RRC).

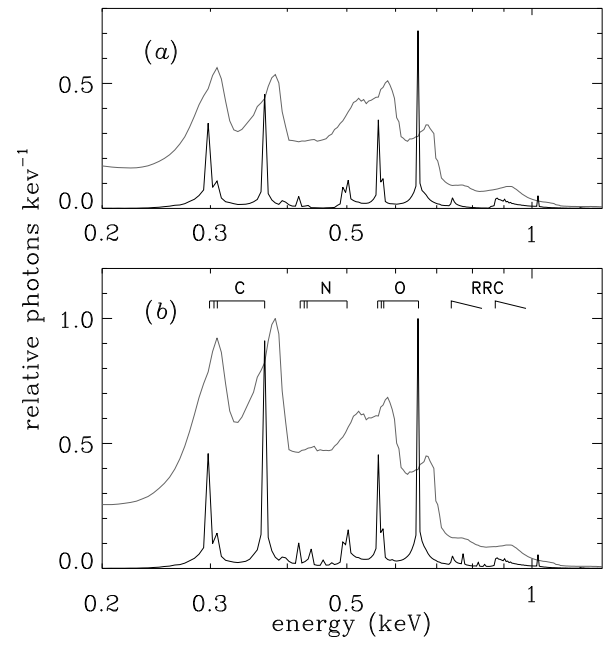

FIG. 4.- Emergent spectra resulting from the production in the disk of $\operatorname{Ly} \alpha$, $\mathrm{He} \alpha$, and RRC photons of $\mathrm{H}-$ and He-like $\mathrm{C}, \mathrm{N}, \mathrm{O}$, and Ne. Panel $(a)$ ignores recombination emission following photoionization, while panel $(b)$ accounts for this emission. Black curves are the spectra in the fluid frame and gray curves are the spectra at infinity at $i=35^{\circ}$. Energies of various Ly $\alpha$ and He $\alpha$ lines and the O RRCs are indicated.

\section{SUMMARY AND CONCLUSIONS}

By means of a Monte Carlo code that accounts for Compton scattering and photoabsorption followed by recombination, we have investigated the radiation transfer of $\operatorname{Ly} \alpha, \operatorname{He} \alpha$, and RRC photons of $\mathrm{H}$ - and He-like $\mathrm{C}, \mathrm{N}, \mathrm{O}$, and Ne produced in the photoionized atmosphere of a relativistic black hole accretion disk. We find that the conversion efficiencies of these photons into lower-energy lines and RRCs are high, and that accounting for this reprocessing significantly (by factors of $21 \%$ to $105 \%$ ) increases the flux of the $\mathrm{Ly} \alpha$ and $\mathrm{He} \alpha$ emission lines of $\mathrm{H}-$ and He-like $\mathrm{C}$ and $\mathrm{O}$ escaping the disk atmosphere. These lines dominate the observed spectrum (Fig. $4 b$ ) because the relative emissivities of these ions are high; the relative photoelectric opacities of these ions are high (Fig. 1), so photons with energies of 0.39 to $2.4 \mathrm{keV}$ are preferentially absorbed by $\mathrm{C}$ and $\mathrm{O}$ and converted into line and RRC photons (Fig. 2); and RRC photons are converted with high efficiencies into lines (Fig. 3). The relative strength of the C VI Ly $\alpha$ line is particularly high in our model because it is fed strongly by all higher- $Z$ ions.

Our model spectrum appears to differ from the Chandra HETG and XMM-Newton RGS spectra of MCG -6-30-15 and Mrk 766 in having too much flux in the $\mathrm{C} \mathrm{V}$ and $\mathrm{O}$ VII $\mathrm{He} \alpha$ lines and too little flux in the N VII Ly $\alpha$ line. The relative strength of the $\mathrm{C} \mathrm{V}$ and $\mathrm{O}$ VII He $\alpha$ lines may not be of concern, since it should be possible to weaken these lines by increasing the mean ionization level of the disk atmosphere: in their photoionized constant-density disk models, BRF find that the Ly $\alpha$ lines dominate the soft X-ray spectrum for ionization parameters $\xi \gtrsim 500$. In contrast, the relative weakness of the $\mathrm{N}$ VII Ly $\alpha$ line may be of concern. BRF find that the strength of this line can be increased simply by increasing the $\mathrm{N}$ abundance. However, we note that if the opacity from 0.55 to 0.67 $\mathrm{keV}$ comes to be dominated by $\mathrm{N}^{5+}$, it and not $\mathrm{C}$ will capture the $\mathrm{O}$ VIII Ly $\alpha$ and $\mathrm{O}$ VII $\mathrm{He} \alpha$ photons, leading to an increase in the N VI He $\alpha$ line strength. Sako (2003) has proposed an alternate way to increase the $\mathrm{N}$ VII $\mathrm{Ly} \alpha$ line strength by a Bowen-like fluorescence mechanism, wherein O VIII Ly $\alpha$ photons pump the $\mathrm{N}$ VII Ly $\zeta$ transition, and the resulting $\mathrm{N}$ VII Ly $\zeta$ photons are converted into N VII Ly $\alpha$ photons via line opacity. We are not yet able to test this mechanism quantitatively, but we expect that it will not be particularly efficient in an irradiated disk atmosphere, since the O VIII Ly $\alpha$ photons are produced in a layer where the $\mathrm{O}^{7+}$ ionization fraction is low and the $\mathrm{N}^{6+}$ ionization fraction is nearly zero. Only those O VIII Ly $\alpha$ photons that are directed down into the disk and survive passage through the $\mathrm{O}^{7+}$ layer will have a chance of being converted into N VII Ly $\alpha$ photons.

In its current form, our Monte Carlo code treats in considerable detail many of the physical processes that affect the transfer of X-ray photons in the photoionized atmosphere of a relativistic black hole accretion disk, but it does not yet follow photons along geodesics and it does not yet account for line opacity. When these processes are included in the code, we will be able to directly calculate the disk spectrum observed at a given inclination at infinity and study the Bowen fluorescence mechanism, the radiation transfer of higher-order Lyman lines, and many other physical processes that affect the X-ray spectra of AGN disks.

The authors acknowledge with gratitude numerous helpful discussions with Masao Sako and Diego Torres. This work was performed under the auspices of the U.S. Department of Energy by University of California Lawrence Livermore National Laboratory under contract No. W-7405-Eng-48.

\section{REFERENCES}

Ballantyne, D. R., Ross, R. R., \& Fabian, A. C. 2002, MNRAS, 336, 867, BRF Bardeen, J. M., Press, W. H., \& Teukolsky, S. A. 1972, ApJ, 178, 347

Branduardi-Raymont, G., Sako, M., Kahn, S. M., Brinkman, A. C., Kaastra, J. S., \& Page, M. J. 2001, A\&A, 365, L140

Jimenez-Garate, M. A., Raymond, J. C., \& Liedahl, D. A. 2002, ApJ, 581, 1297

Jimenez-Garate, M. A., Raymond, J. C., Liedahl, D. A., Mauche, C. W. 2003, in preparation

Krolik, J. H., McKee, C. F., \& Tarter, C. B. 1981, ApJ, 249, 422

Laor, A. 1991, ApJ, 376, 90

Lee, J. C., Ogle, P. M., Canizares, C. R., Marshall, H. L., Schulz, N. S., Morales, R., Fabian, A. C., \& Iwasawa, K. 2001, ApJ, 554, L13

Mason, K. O., et al. 2003, ApJ, 582, 95

Miller, K. A., \& Stone, J. M. 2000, ApJ, 534, 398

Page, D. N. \& Thorne, K. S. 1974, ApJ, 191, 499

Raymond, J. C. 1993, ApJ, 412, 267
Riffert, H., \& Herold, H. 1995, ApJ, 450, 508

Ross, R. R., Fabian, A. C., \& Brandt, W. N. 1996, MNRAS, 278, 1082

Rybicki, G. B., \& Lightman, A. P. 1979, Radiative Processes in Astrophysics (New York: Wiley)

Sako, M. 2003, ApJ, in press [astro-ph/0305514]

Sako, M., Kahn, S. M., Branduardi-Raymont, G., Kaastra, J. S., Brinkman, A. C., Page, M. J., Behar, E., Paerels, F., Kinkhabwala, A., Liedahl, D. A., \& den Herder, J. W. 2003 ApJ, 596, 114

Sako, M., Liedahl, D. A., Kahn, S. M., \& Paerels, F. 1999, ApJ, 525, 921

Shakura, N. I, \& Sunyaev, R. A. 1973, A\&A, 24, 337

Thorne, K. S. 1974, ApJ, 191, 507

Turner, A. K., Fabian, A. C., Vaughan, S., \& Lee, J. C. 2003, MNRAS, 346, 833

Verner, D. A., \& Yakovlev, D. G. 1995, A\&AS, 109, 125 
University of California

Lawrence Livermore National Laboratory

Technical Information Department

Livermore, CA 94551

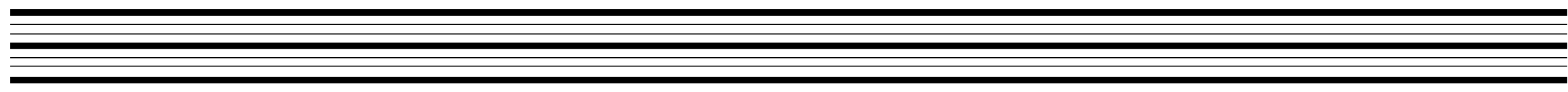

